\title{
Cultura de la información en el contexto educativo universitario: aportes teóricos
}

\author{
Dora Caudillo* \\ Socorro Encinas-Grijalva**

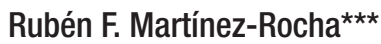 \\ Jesús Lau****
}

Artículo recibido:

27 de mayo de 2021

Artículo aceptado:

7 de septiembre de 2021

Artículo de investigación

\section{Resumen}

La cultura de la información (CI) surge de crear flujos de información para optimizar procesos comunicativos dentro de una organización, paralelo al desarrollo de tecnologías de información y comunicación y a una serie de contribuciones de distintas disciplinas que dieron origen a este fenómeno. El proceso de analizar y comprender su complejidad remite a su estudio desde diversas perspectivas, dada la multidisciplinariedad que la caracteriza. El objetivo de esta investigación es contribuir con aportes teóricos al campo de estudio

* Colegio de Ciencias Sociales y Humanidades, CETYS Universidad, Tijuana, B. C., México dora.caudillo@cetys.mx

** Escuela de Administración y Negocios, CETYS Universidad, Tijuana, B. C., México socorro.encinas@cetys.mx

*** Dirección de Bibliotecas, CETYS Universidad, Tijuana, B. C., México ruben.martinez@cetys.mx

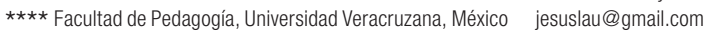
INVESTIGACIÓN BIBLIOTECOLÓGICA, vol. 36, núm. 90, enero/marzo, 2022, México, ISSN: 2448-8321 pp. 133-149 
de la CI en el ámbito educativo, específicamente desde el nivel superior y desde el enfoque del acceso y uso de información. El análisis conceptual se realizó con metodología de corte cualitativo, la cual permitió identificar elementos semánticos generados desde el contexto organizacional y cómo éstos se insertaron en el entorno educativo. Se utilizó el método de comparación constante (MCC) a partir de la integración teórica del campo intelectual creador (CIC). Los resultados confirman una relación de elementos que transitaron del campo organizacional a los procesos de aprendizaje de educación superior, donde a dichas instituciones les corresponde implementar estrategias para el desarrollo de habilidades en el acceso y uso de información como componentes vitales de CI.

Palabras clave: Cultura de la Información; Capital Intelectual; Cultura Organizacional; Educación Superior; Habilidades Informacionales; Gestión de la Información

Information Culture in higher education context: theoretical contributions

Dora Caudillo, Socorro Encinas-Grijalva, Rubén F. Martinez-Rocha and Jesús Lau

\section{Abstract}

Information culture (IC) arises from information flows to optimize communication processes within an organization, parallel to development of information and communication technology and a series of contributions from different disciplines that gave rise to the phenomenon. Process of analyzing and understanding its complexity refers to its study from different perspectives; given the multidisciplinarity that characterizes it. The purpose of this paper is to provide theoretical contributions to the field of study on IC in educational field, specifically from higher education level and approach of access and use of information. Conceptual analysis was carried out with qualitative methodology, which allowed to identify semantic elements generated from organizational context and how these were inserted in educational environment. Constant comparison method (MCC) was used from theoretic integration of 
creative intellectual field (CIC). Results confirm some elements that went from organizational to higher education learning processes, where these institutions are responsible for implementing strategies for development of skills in access and use of information as vital components of IC. Additionally, value of information as intellectual capital is argued and it is established as a conclusion that, implementation of IC emerges from generation of intellectual capital and the fact that this is monitored and measured or quantified in systematic way, it can be affirmed that there is a consolidated IC.

Keywords: Information Culture; Intellectual Capital; Organizational Culture; Higher Education; Information Literacy; Information Management

\section{INTRODUCCIÓN}

Fl término cultura de la información fue utilizado por primera vez en 1987 Lpor Ginman, quien estableció que "cultura de la información se correlaciona positivamente con un desempeño comercial [organizacional] estrechamente relacionado con actividades, actitudes y culturas comerciales que generan resultados exitosos" (1987: 104). Sin embargo, se puede identificar una cultura de la información (CI) en cualquier organización donde la información sea la base para la toma de decisiones apoyadas por las tecnologías de información (Virkus y Salman, 2020).

El concepto de CI se ha estudiado principalmente desde un contexto empresarial en el que se identifica que las organizaciones buscan cumplir objetivos y metas donde sobresalen comportamientos relacionados con el uso de información, los cuales surgen de normas preestablecidas por cada organización. En ese sentido, se han reconocido diversas culturas de la información (Oliver, 2011) como resultado del comportamiento organizacional, las cuales retratan valores y actitudes propias de cada organización en relación a la gestión de la información.

Desde el ángulo epistemológico, la CI se vincula con diversas áreas disciplinares, tales como bibliotecología, ciencias de la información, sociología, psicología y ciencias administrativas; sin embargo, las diferentes definiciones, enfoques y modelos indican que este concepto funciona en conjunto con otras disciplinas (Deja y Wójcik, 2020), lo cual condiciona que la comprensión conceptual de CI requiere un análisis multidisciplinar. 
Existen ciertos componentes de CI que han emigrado de lo organizacional a lo educativo, relacionados con el valor de la información, lo que ha permitido identificar estándares sobre el comportamiento de la información para que las instituciones educativas puedan desarrollar una cultura informativa vinculada con la formación de profesionales competentes. En ese sentido, Abilova et al. (2019: 8) mencionan que los ambientes educativos hacen posible una mejor formación y desarrollo de CI. Para ello se requiere de un modelo de educación tecnopedagógico para la generación y fomento de sociedades de la información y del conocimiento como consecuencia de la inserción de tecnologías que faciliten el aprendizaje (Pérez-Zúñiga et al., 2018).

El objeto de esta investigación es contribuir con aportes teóricos a partir de la identificación de dichos componentes vinculados con el valor y comportamiento de la información y su relación al campo de estudio de la CI en el ámbito educativo, específicamente desde la educación superior.

\section{Cultura de LA INFORMación EN EL ENTORNO EDUCATivo}

El progreso científico y tecnológico es una constante que demanda cambios fundamentales en la educación, donde hay nuevos valores e ideas sobre el mundo, lo que conduce al nuevo hábitat natural del hombre moderno (Bozieva, Zhurtov y Bosieva, 2018). Por lo tanto, una de las principales características de la sociedad de la información es la disponibilidad de recursos de información, de las tecnologías de información y la presencia de una estructura de información acorde al entorno.

Si la educación es construida sobre la base de una CI, será necesario que el individuo cuente con la capacidad para percibir, crear, analizar y evaluar información con el fin de generar habilidades y asimilar conocimientos para comprender el contexto sociocultural y político moderno. No es casualidad que son las instituciones de educación superior (IES) las que han comenzado a focalizar su interés en CI como parte de su estructura académica, implementando acciones específicas como inversión en infraestructura tecnológica, diseño de programas curriculares, contratación de personal especializado, y capacitación a personal académico y administrativo. Dichas acciones permiten fortalecer el ambiente de CI como un elemento diferenciador en la formación de estudiantes.

La identificación de saberes digitales en universitarios (Ramírez y Casillas, 2015, en Salado-Rodríguez y Ramírez-Martinell, 2018) ha sido una propuesta prioritaria para que las IES tomen decisiones relacionadas con gastos sobre adquisición de recursos tecnológicos, diseño e implementación de planes y 
programas. En ese sentido, Bourdieu (2008) señala que los usos y aplicaciones de herramientas tecnológicas que se institucionalizan en alguna organización podrían estar determinados por el habitus, que origina prácticas individuales y colectivas que permiten comprender cómo se implementa en la práctica el uso de tecnología digital en los individuos vinculados al marco institucional, que finalmente son quienes generarán una transformación en el proceso educativo mediado por TIC (Salado-Rodríguez, Velázquez García y Ochoa Landín, 2016).

En esta dimensión, Casillas Alvarado, Ramírez-Martinell y Ortiz (2014) denominan "capital tecnológico" (KT) al hecho de integrar TIC a los procesos educativos desde el punto de vista técnico o instrumental, y consideran que los aspectos sociológicos influyen en la apropiación del estudiante, que es el objetivo final de toda institución educativa. Esta propuesta es un camino adecuado para el análisis de distintos niveles de adopción, uso y disposición que tienen los actores universitarios hacia la tecnología.

Para lograr el objetivo de este estudio, es necesario identificar los elementos que conformaron a la CI desde su origen en el ámbito organizacional y cómo transitaron al entorno educativo. Para ello, se utilizó una metodología que permitiera hacer un análisis documental para categorizar los elementos que la conforman.

\section{Metodología}

El análisis se sustentó en la teoría de Corbin y Strauss (2014) para desarrollar dos operaciones específicas: por un lado, formular preguntas para aumentar el entendimiento teórico del núcleo disciplinar tratado y, por otro, hacer comparaciones entre la propia obra de Bourdieu y los contextos, estructuras, procesos y consecuencias directas al campo de la investigación de la CI. Ambos procedimientos constituyeron el proceso analítico esencial del método de comparación constante (MCC). El valor de la metodología interpretativa, como lo expresa la teoría fundamentada, radica en su capacidad no sólo de generar teoría, sino también de fundamentarla en los datos y en la realidad (León-Duarte, 2019).

El análisis conceptual de CI se basó en una metodología cualitativa mediante técnicas y procedimientos de comparación constante (MCC) a partir de la integración teórica del campo intelectual creador (CIC) de la teoría de los campos de Bourdieu (2008). Esto permitió establecer relaciones y conexiones conceptuales para organizar un esquema explicativo de carácter teórico conceptual, en búsqueda de un primer acercamiento al origen del concepto de CI. 
La revisión bibliográfica permitió identificar dos elementos fundamentales: los documentos elegidos y el resultado de la agregación/interpretación de los mismos. Por tanto, una revisión bibliográfica equivale a una investigación en la cual los documentos seleccionados son los datos primarios y su agregación/interpretación el método de análisis (Codina, 2018: 6).

El estudio se realizó a partir de 98 publicaciones generadas entre 1987 y 2020, todas referentes a CI, con el objetivo de distinguir aportes teóricos que permitan identificar una $\mathrm{CI}$ en el entorno educativo del nivel superior. Para ello se examinaron e identificaron similitudes y diferencias de aplicación entre la categoría conceptual de CI y el campo de investigación para poder distinguir entre constructos, factores y propiedades de carácter teórico-conceptual. Finalmente, se clasificaron las categorías, factores e indicadores como propiedades del CI dentro de un marco intelectual creador.

Los criterios de búsqueda y selección de la literatura se establecieron a partir de las palabras clave cultura de la información, en español, e information culture, en inglés. Las bases de datos utilizadas fueron Springer, EBSCO, Emerald, Scopus y Google Académico, ya que son las bases de datos de acceso institucional. El procedimiento de búsqueda resultante se limitó a los criterios de inclusión, cultura de la información y relevancia la CI en educación superior.

A partir de lo anterior, se planteó como variable principal la educación superior, en la cual se analizó la CI, entendiendo la educación superior como el aprendizaje que se construye después del bachillerato o equivalente y comprende educación normal, tecnológica y universitaria e incluye carreras profesionales cortas y estudios encaminados a obtener los grados de licenciatura, maestría y doctorado, así como cursos de actualización y especialización (SEP, 2021).

\section{Presentación y ANÁLISIS DE RESUltados}

Los resultados obtenidos buscan identificar los componentes de este concepto en el entorno específico de educación superior, así como incidir en el cuerpo epistémico disciplinar. Las investigaciones que han definido el comportamiento de CI se han contextualizado en el ámbito empresarial, cuyos enfoques son de relevancia para extrapolar procesos y experiencias al ámbito educacional.

Uno de los objetivos esenciales de las IES es facilitar la transformación de jóvenes estudiantes en profesionistas competentes; este proceso requiere de una cultura informativa, sin la cual difícilmente tendrán las capacidades 
que demanda el mercado laboral. En esta función social educativa, un agente transformador potencial de los modelos educativos son las TIC, a través de las cuales se transfieren grandes volúmenes de información que permiten que el conocimiento trascienda del marco institucional, lo cual confirma que el saber es un medio de desarrollo con el objetivo de producir conocimiento con un amplio sentido social (Pescador Vargas, 2014).

En el escenario educacional de instituciones de vanguardia, se corrobora que la característica predominante es el uso de dispositivos digitales para facilitar el aprendizaje, esto consolida un modelo integral de educación, cumpliendo con los objetivos tecnopedagógicos de actualidad (Pérez-Zúñiga et al., 2018). El análisis de literatura realizado como parte de esta investigación confirma lo señalado por estos autores, ya que se identifica que las instituciones educativas idealmente podrían

a) Promover la cultura del uso de TIC por parte de la comunidad académica y estudiantil;

b) Implementar programas de alfabetización digital de mejora continua en función de la enseñanza-aprendizaje, y

c) Apoyarse en el capital intelectual colectivo de la institución.

De acuerdo con el núcleo teórico analizado, no existe un consenso sobre el término de CI. Las diferentes acepciones hacen énfasis en elementos específicos que obedecen a comportamientos distintos de información dependiendo del contexto en el cual se desarrolle. Sin embargo, dos constantes destacan en este análisis (Zheng, 2005). Primero, CI no puede ser "creada" o "establecida", porque siempre ha existido como dimensión de la cultura organizativa. El concepto, según el mismo autor y con el cual se coincide, señala que está arraigada a entornos históricos y sociales, pero evoluciona ya que puede ser cultivada, desarrollada o conformada, sujeta a una gestión adecuada y a una formulación institucional.

Segundo, los sistemas técnicos, aunque podrían ser cruciales, no se consideran constitutivos de CI; sin embargo, la tecnología es parte de los recursos que los seres humanos utilizan para dar forma a su CI, es decir, ésta existe con o sin tecnologías de información. En este estudio se reconoce la presencia de TIC en la comprensión de CI en un entorno de educación superior, considerando a los recursos tecnológicos como un eslabón clave para el acceso a información y conocimiento.

A partir de las obras analizadas, se obtuvo una lista de propiedades básicas para definir particularidades en el análisis de CI, la cual se estableció como categoría de primer orden (Tabla 1). Posteriormente, se determinaron 
factores de segundo orden, los cuales permiten identificar seis categorías a partir del comportamiento de información en ambos entornos de estudio (organizacional y educativo). Por último, se presenta una serie de 15 indicadores descriptivos y predictivos de propiedades de distintos comportamientos que ha presentado la información dentro del contexto de CI.

\begin{tabular}{|c|c|c|}
\hline Categoría de primer orden & Factores de segundo orden & Indicadores \\
\hline $\begin{array}{l}\text { Cultura de información como } \\
\text { campo intelectual creador }\end{array}$ & $\begin{array}{l}\text { 1. Información } \\
\text { 2. Sistemas de información } \\
\text { 3. Alfabetización informacional } \\
\text { 4. Instituciones, organizaciones } \\
\text { y personas } \\
\text { 5. Gestión del conocimiento } \\
\text { 6. Identidad social }\end{array}$ & $\begin{array}{l}\text { 1. Información en contexto empresarial } \\
\text { 2. Flujos de información } \\
\text { 3. Comportamientos y normas } \\
\text { de información } \\
\text { 4. Ecología de información } \\
\text { 5. Normas, valores, creencias } \\
\text { y actitudes en el uso de información } \\
\text { 6. Capital intelectual } \\
\text { 7. Sociedad de información y del } \\
\text { conocimiento } \\
\text { 8. Rol del profesional de información } \\
\text { 9. Políticas públicas sobre el uso de } \\
\text { información } \\
\text { 10. Patrones de comportamiento de la } \\
\text { información } \\
\text { 11. Tecnologías de Información } \\
\text { y Comunicación } \\
\text { 12. Gestión de información } \\
\text { 13. Multidisciplinariedad de Cl } \\
\text { 14. Normas ALFIN } \\
\text { 15. Configuraciones sociales a partir } \\
\text { de información. }\end{array}$ \\
\hline
\end{tabular}

Tabla 1. Descripción de las aportaciones teóricas del campo de Cl como campo intelectual creador Fuente: elaboración propia

Los resultados obtenidos por medio del modelo de MCC permitieron examinar e integrar nuevas relaciones de organización y clasificación de la teoría a partir de un conjunto selectivo de dimensiones sobre $\mathrm{CI}$ en las cuales se identificaron tres categorías en el entorno de educación superior: cultura de la información como capital cultural, información como capital cultural en el ámbito educativo, y sociedad de información y del conocimiento como eje central de CI.

\section{Cultura de la información como capital cultural}

Uno de los primeros hallazgos se relaciona con el uso de TIC debido a que requiere de conocimientos previos para poder acceder a la información. Al 
respecto, Casillas Alvarado, Ramírez-Martinell y Ortiz (2014) mencionan que el KT (capital tecnológico) comprende al conjunto de saberes que la institución educativa reconoce de manera formal durante el proceso de aprendizaje. Su posesión es un atributo que diferencia a los individuos y les permite competir de mejor manera en diversos campos, espacios sociales y laborales.

Esto se relaciona con el babitus digital, que Casillas Alvarado y Ramírez-Martinell (2018: 323) definen como "un conjunto de capacidades y prácticas que los individuos desarrollan en el marco de cultura digital”. Estos autores consideran que el KT se puede apreciar por medio del tiempo de trabajo invertido y los procesos de aprendizaje (formales y no formales).

Asimismo, el KT se puede medir igual que el capital cultural de Bourdieu, y se distinguen en él dos fases:

a) En su estado objetivado, el KT es el conjunto de objetos tecnológicos que son apropiados en su materialidad y en su significado simbólico (inversión en diferentes rubros respecto a los insumos de tecnología). Desde este eje, el capital incorporado se constituye por los saberes que tiene el estudiante relacionados con las TIC (Salado-Rodríguez y Ramírez-Martinell, 2018).

b) En su estado institucionalizado, el KT se refiere al conjunto de títulos, diplomas y certificados cuyo valor simbólico (factor institución, grado de prestigio) valida, instituye y reconoce saberes, conocimientos y habilidades, y define un status jerárquico por el tipo de conocimiento. Se mide en términos del número de cursos, diplomas y certificados (Casillas Alvarado, Ramírez-Martinell y Ortiz, 2013; 2014).

La identificación de saberes digitales de los universitarios, dependiendo de sus campos disciplinarios (Casillas Alvarado y Ramírez-Martinell, 2021: 172192), ha sido propuesta como tarea prioritaria para que las IES tomen decisiones informadas sobre orientación del gasto, reestructuración de planes, programas y capacitación de profesores.

Es importante observar al fenómeno como una apropiación de tecnología digital, entendida como aprehensión de prácticas que derivan en un uso culturalmente organizado de tecnología (Núñez et al., 2013), donde el punto de coincidencia de los autores es disponer de dispositivos tecnológicos y ser hábiles en el uso de software como medios para el aprendizaje.

Analizar los usos y prácticas de TIC desde la perspectiva bourdiana (Casillas Alvarado y Ramírez-Martinell, 2018) contribuye a la conceptualización teórica de capitales culturales, que permite la discusión acerca de cómo se perciben y se utilizan las TIC por parte de los diferentes actores que conforman las 
IES en la preparación y desarrollo de estudiantes y futuros profesionistas. El uso de TIC en IES no es una cuestión de carácter solamente tecnológico, sino que también está relacionado con aspectos sociales y culturales que implican una serie de suposiciones y conceptos, pero, sobre todo, de habilidades que adquieren los universitarios durante su formación profesional.

\section{Información como capital intelectual en el ámbito educativo}

Una CI altamente desarrollada se correlaciona positivamente con el desempeño exitoso de flujos de información, según Ginman (1987), por lo que se puede asumir que existe una gran conexión entre transformaciones de recursos intelectuales y materiales que resultan en una CI caracterizada por una conexión entre el individuo y el producto intelectual que genera, gestionado por la empresa u organización para obtener un producto con valor.

El capital intelectual incluye a los activos intangibles y activos de conocimiento, obteniendo como resultado final la asociación de factores humanos, estructurales, relacionales y sociales que agrupa una organización, relacionados con el conocimiento de sus miembros y aplicados al trabajo que proporcione una ventaja competitiva a la empresa o institución. En este sentido, destaca lo siguiente (Vega Falcón, 2017):

a) Activos intangibles: propiedad intelectual, desarrollo de patentes, desarrollo de software, capital humano (conocimiento y habilidades de docentes y profesores) y diseños curriculares.

b) Activo tangible: capital estructural (infraestructura tecnológica).

Por su parte, el talento humano constituye uno de los esenciales activos del ámbito educativo, lo que representa el valor más significativo. Identificar y medir el capital intelectual tiene como particularidad hacer visible el activo que objetivamente crea más valor. Para lograrlo, Vega Falcón (2017) distingue tres elementos, que para fines de esta investigación se han vinculado con los actores principales pertenecientes a un contexto de enseñanza-aprendizaje:

a) Competencias, expresadas en conocimientos, capacidades, y talento (profesores, maestros, docentes, instructores).

b) Actitudes, que se convierten en conductas, motivaciones, actuaciones y ética de personas (estudiantes).

c) Vitalidad intelectual, capaz de generar valor para la organización, proporcionalmente a la aplicación de nuevos conocimientos que posibilitan convertir las ideas en productos y servicios (institución educativa). 
El hallazgo más relevante de esta investigación es el capital intelectual, que es uno de los factores clave que han emigrado de lo organizacional a lo educativo y que se considera como el componente clave de CI en la educación. Cada vez es mayor el número de IES que le dan importancia al capital intelectual; sin embargo, las distintas iniciativas de medición se desarrollan dentro de actividades generales de las propias universidades, sin que exista algún tipo de coordinación con un programa específico de capital intelectual. Axtle-Ortiz y Acosta-Prado (2017) señalan que la evaluación de riqueza intangible de las organizaciones frecuentemente se realiza de forma fragmenta$\mathrm{da}$, reflejando ausencia o deficiencia de una estrategia específica para medirlo.

\section{Sociedad de información y del conocimiento como eje central de CI}

Sociedad de información (SI) y sociedad del conocimiento (SC) son dos expresiones que escasamente se encontraron en la literatura analizada en relación a CI; particularmente se identificaron en aquellos textos donde se vincula la información con procesos de comunicación mediados por tecnología en ambientes escolares. Los textos que plantean el fenómeno de CI relacionado con el entorno organizacional prácticamente no mencionaron SI ni SC. Para los fines de esta investigación, centrada en el campo educativo, SI y SC no sólo se refieren al uso de dispositivos digitales para facilitar el acceso, sino también lograr un aprendizaje y consolidar un modelo integral de educación que cumpla con objetivos tecnopedagógicos y genere capital intelectual.

En relación a ello, la introducción de tecnologías en las aulas y el incremento de cursos en línea han abierto nuevos horizontes para mejorar la calidad de educación y han incidido en la transformación de modelos educativos que se basan en infraestructura tecnológica y en internet para procesar y transmitir información (Pérez-Zúñiga et al., 2018). Todo esto se considera parte del indicador "acceso a información” (núm. 14 en Tabla 1), donde TIC son un componente esencial en el estudio de CI.

Gracias al desarrollo tecnológico, cada vez es más fácil acceder a información, de tal manera que todos los contextos elementales de una sociedad están involucrados. Según Verón Lassa (2016), esto implica un cambio en el alcance del saber con un amplio sentido social, por lo que el conocimiento no se limita únicamente al ámbito académico; el saber es un medio de desarrollo que facilita el acceso e intercambio de información y datos.

La SC como un indicador de CI (núm. 7 en Tabla 1) contribuye a buscar la expresión científica y tecnológica y permite adquirir una ventaja competitiva en el desarrollo y generación de oportunidades para el bienestar y progreso humano. 
En síntesis, en concordancia con Pérez-Zúñiga et al. (2018), las SI y SC son condiciones necesarias para impulsar una educación de calidad. Para esto, se requiere que la estabilidad estructural y tecnológica de instituciones educativas se constituya en una educación pertinente, centrada en el progreso y modernidad, con el fin de responder a requerimientos de la sociedad actual. Para impulsar dichas capacidades en el acceso a información, los individuos requieren desarrollar habilidades específicas que les permitan utilizar información de manera óptima, indicador que corresponde al tercer factor del segundo orden (Alfabetización informacional) y al indicador núm. 14 en Tabla 1 (Normas ALFIN).

\section{Alfabetización informacional (ALFIN) como proceso para el desarrollo de habilidades y competencias informacionales}

Adicional a las tres categorías presentadas, es necesario destacar la presencia de ALFIN como elemento fundamental para que pueda desarrollarse una CI consolidada en el ámbito educativo. En relación a ello, el paradigma de ALFIN propone un conjunto de herramientas y técnicas que inciden en la madurez e independencia del estudiante para localizar, evaluar, sintetizar, comunicar y obtener conocimiento en un ambiente colaborativo, mientras que a los docentes les corresponde promover competencias informacionales (Moreno-Guerrero et al., 2020).

La inserción de ALFIN en las instituciones educativas, con el propósito de formar una CI, requiere de la participación de todos los entes que participan en el ciclo de formación-aprendizaje. Entre los principales argumentos que hacen de ALFIN un factor determinante para el desarrollo de CI en las instituciones de educación; Pirela Morillo y Cortés Vera (2014), Moreno-Guerrero et al. (2020), y Righetto y Vitorino (2020) resaltan las siguientes:

1. Diseñar políticas y planes institucionales que contribuyan de manera integradora a la SC.

2. Focalizar al sistema bibliotecario como soporte de las actividades curriculares.

3. Mejorar el aprovechamiento de innovaciones educativas.

4. Desarrollar métodos pedagógicos innovadores.

5. Promover la enseñanza transversal del docente, así como prácticas que incidan en la calidad educativa.

6. Aprovechar los avances tecnológicos para un mejor acceso a la educación y en consecuencia la formación de ciudadanos más críticos y activos en una sociedad globalizada. 
Dado lo anterior, se ratifica la utilidad de ALFIN como constructo habilitador de estudiantes y docentes, especialmente para generar, compartir y asimilar conocimiento a partir de información. El conjunto de habilidades y competencias que se forman en este proceso es consecuencia de la estrategia implementada por IES en función de estructurar una CI orgánica, endógena y dinámica, que sea sostenible, mientras que la información debe ser el núcleo rector del aprendizaje continuo en la institución educativa, coherente con prácticas mediáticas actuales y con el entorno digital.

\section{Conclusiones}

La CI está profundamente arraigada en entornos históricos y sociales; evoluciona constantemente, se fortalece con el desarrollo tecnológico, sujeta a una gestión y una formulación institucional adecuadas. En este sentido, a partir de aportes teóricos, se confirma que es necesario su abordaje desde un enfoque multidisciplinar. En este proceso intervienen valores sociales y patrones de comportamiento relacionados con recopilación, interpretación y utilización de información.

El concepto de CI en el entorno educativo implica una mejora de capacidades del individuo dentro de un proceso de enseñanza-aprendizaje. Las instituciones educativas en el escenario de SI y SC deben modernizar los modelos de aprendizaje y recursos tecnológicos que fomenten el conocimiento. Este cambio debe tener como punto central brindar a los alumnos las oportunidades para que desarrollen y promuevan la investigación en la sociedad actual. Deben acentuar los métodos formativos enfocados en conocimiento, información y tecnología; así, la mejora educativa exige procesos pedagógico-formativos como valores esenciales para lograr cambiar la conducta humana, de modo que las personas involucradas alcancen un crecimiento exponencial de sus funciones cognitivas.

Es importante señalar que la incorporación de TIC a la educación y el acceso a información requiere de acciones para cultivar saberes que se transformen en conocimiento para conseguir soluciones a los problemas cotidianos. Las universidades deben contribuir a la SI y la SC a partir del progreso y la modernidad y en coherencia con los requerimientos de la sociedad actual.

De acuerdo a factores identificados en este estudio, destaca como hallazgo el concepto de capital intelectual, el cual contempla la integración de TIC desde el punto de vista técnico e instrumental y considera aspectos sociológicos que influyen en la apropiación del conocimiento en estudiantes; esto da como resultado la formación de seres humanos analíticos, reflexivos, capaces 
de aprender y comunicarse de manera efectiva en relación con la información y en una sociedad más desarrollada.

En concordancia con las investigaciones de Yong Amaya et al. (2017), se puede considerar entonces al capital intelectual como un factor innovador en las universidades, a partir de la vinculación del conocimiento con las necesidades sociales por medio de activos intangibles para crear una ventaja competitiva capaz de impulsar transformaciones de fondo. Utilizar los elementos del capital intelectual en las universidades contribuye a identificar y valorar criterios ya existentes, pero no controlados, que garantizan la calidad de la educación superior, y a mejorar los niveles de excelencia y la correcta gestión de estos intangibles que a su vez permitan la toma de decisiones eficientes.

Finalmente, es conveniente que las IES comiencen a medir su capital intelectual y monitorear aquellos componentes que han sido detectados con un alto impacto en la generación de valor, pues allí se encuentran ventajas competitivas. En este orden de ideas es necesario realizar estudios focalizados para conocer el uso e impacto de TIC en IES. El camino hacia el desarrollo social y económico debe partir de la información como capital intelectual que va dirigido a SI y SC, ambas como eje de una cultura de información consolidada.

Agradecimientos

Los autores expresan su amplio y cumplido agradecimiento a los integrantes de la empresa Habilis I\&CC por el valioso apoyo otorgado en la construcción y revisión de este artículo.

\section{REFERENCIAS}

Abilova, Oryngul, Aynash Kudysheva, Bagit Kalimukhasheva, Aigul Tulegenova, Zhanna Kulbatyrova y Barshagul Stamgaliyeva. 2019. "The Phenomenon of the Logical-Information Culture of the Individual in the Context of Global Changes in Modern Society”. Dilemas Contemporáneos: Educación, Política y Valores 7 (1): 1-21. https://www.dilemascontemporaneoseducacionpoliticayvalores.com/index. $\mathrm{php} /$ dilemas/article/view/1729/1359

Axtle-Ortiz, Miguel Ángel y Julio César Acosta-Prado. 2017. "Medición y gestión del capital intelectual en las instituciones de educación superior”. Dimensión empresarial 15 (2): 103-115. https://doi.org/10.15665/rde.v15i2.1306

Bourdieu, Pierre. 2008. El sentido práctico. España: Siglo XXI de España Editores.

Bozieva, Asiyat M., Artur V. Zhurtov y Mariam M. Bozieva. 2018. "Modern Information Society and its Impact on the Education”, en 2018 IEEE International Conference "Quality Management, Transport and Information Security, Information Technologies" (ITEQMEIS), 587-588. Nalchik, Rusia: IEEE. 
Casillas Alvarado, Miguel Ángel, Alberto Ramírez-Martinell y Juan Carlos Ortega Guerrero. 2016. "Afinidad tecnológica de los estudiantes universitarios". Innovación educativa 16 (70): 151-175.

http://www.scielo.org.mx/scielo.php?script=sci_arttext\&pi$\mathrm{d}=$ S1665-26732016000100151\&lng=es\&tlng=es

Casillas Alvarado, Miguel Ángel, Alberto Ramírez-Martinell y Verónica Ortiz. 2013. "El capital tecnológico", en Memorias del XII Congreso Nacional de Investigación Educativa, 1-10. Guanajuato, México.

Casillas Alvarado, Miguel Ángel, Alberto Ramírez-Martinell y Verónica Ortiz. 2014. "El capital tecnológico una nueva especie del capital cultural. Una propuesta para su medición”, en Háblame de TIC: Tecnología Digital en Educación Superior, compilado por Alberto Ramírez Martinell y Miguel Ángel Casillas Alvarado, 2338. Córdoba, Argentina: Editorial Brujas.

https://www.uv.mx/personal/mcasillas/files/2016/05/20.pdf

Casillas Alvarado, Miguel Ángel y Alberto Ramírez-Martinell. 2021. Saberes digitales en la educación. Una investigación sobre el capital tecnológico incorporado de los agentes de la educación. Córdoba: Brujas.

https://www.uv.mx/personal/mcasillas/files/2021/02/Libro.pdf

Casillas Alvarado, Miguel Ángel y Alberto Ramírez-Martinell. 2018. "El babitus digital: una propuesta para su observación”, en Pierre Bourdieu en la sociología latinoamericana: el uso de campo y babitus en la investigación, coordinado por Roberto Castro y Hugo José Suárez, 317-341.

http://hugojosesuarez.com/ArticulosPDFs/LibrosCoordinados/2018\%2C\%20

Bourdieu\%20en\%20la\%20sociologia-\%20Libro\%20completo-Suarez\%20 y\%20Castro.pdf

Codina, Lluís. 2018. Revisiones bibliográficas sistematizadas: procedimientos generales y Framework para ciencias humanas y sociales. Barcelona: Universitat Pompeu Fabra.

Corbin, Juliet y Anselm Strauss. 2014. Basics of qualitative research: Techniques and procedures for developing grounded theory. USA: Sage Publications.

Deja, Marek y Magdalena Wójcik. 2020. "Information culture and academic empowerment: Developing a collective mindfulness strategy for embedded librarianship". The Journal of Academic Librarianship 47 (2). https://doi.org/10.1016/j.acalib.2020.102276

Ginman, Mariam. 1987. "Information culture and business performance”. Iatul Quarterly, The 12th Biennial Conference 2 (2) (Åbo Akademi University): 92-106. http://docs.lib.purdue.edu/iatul/1987/papers/15

León-Duarte, Gustavo Adolfo. 2019. "Aportes teóricos a la investigación del campo periodístico. Sentidos y significados desde el Campo Intelectual Creador". Intercom: Revista Brasileira de Ciências da Comunicação 42: 41-59.

https://doi.org/10.1590/1809-5844201932

Moreno-Guerrero, Antonio-José, Nuria Miaja-Chippirraz, Ana Bueno-Pedrero y Laura Borrego-Otero. 2020. "El área de información y alfabetización informacional de la competencia digital docente”. Revista Electrónica Educare 24 (3): 521-536.

https://doi.org/10.15359/ree.24-3.25 
Núñez Urbina, Alicia Angélica, Elizabeth Cabrera Mendoza, Alejandra Isabel Ledezma Peralta, Hugo Moreno Reyes, Juan Carlos Valdés Godínes y Aurora Adriana Lugo García. 2013. "Apropiación social de las TIC y políticas educativas en la educación superior tecnológica”. Pistas Educativas: 6-16.

http://pistaseducativas.itc.mx/wp-content/uploads/2013/06/1-NUNEZ-PE-101-6-16.pdf

Oliver, Gillian. 2011. Organisational culture for information managers. Oxford, UK: Elsevier. https://doi.org/10.1533/9781780632759

Pérez-Zúñiga, Ricardo, Paola Mercado Lozano, Mario Martínez García, Ernesto Mena Hernández y José Ángel Partida Ibarra. 2018. "La sociedad del conocimiento y la sociedad de la información como la piedra angular en la innovación tecnológica educativa”. RIDE. Revista Iberoamericana para la Investigación y el Desarrollo Educativo 8 (16): 847-870.

https://www.redalyc.org/articulo.oa?id=498159332036

Pescador Vargas, Beatriz. 2014. “¿Hacia una sociedad del conocimiento?” Revista MED 22, (2): 6-7.

http://www.scielo.org.co/scielo.php?script=sci_arttext\&pid=S0121-52562014000200001

Pirela Morillo, Johann, y José de Jesús Cortés Vera. 2014. "El desarrollo de competencias informacionales en estudiantes universitarios: Experiencia y perspectivas en dos universidades latinoamericanas". Investigación bibliotecológica 28 (64): 145-172. http://www.scielo.org.mx/scielo.php?script=sci_arttext\&pi$\mathrm{d}=\mathrm{S} 0187-358 \mathrm{X} 2014000300008$

Righetto, Guilherme Goulart y Elizete Vieira Vitorino. 2020. "A competência em informação como movimento de inovação social”. Investigación bibliotecológica 34 (82): $29-52$.

https://doi.org/10.22201/iibi.24488321xe.2020.82.58080

Salado-Rodríguez, Lilian Ivetthe, Mario Alberto Velázquez García y Reyna Isabel Ochoa Landín. 2016. "La apropiación de las TIC en los estudiantes universitarios: una aproximación desde sus habitus y representaciones sociales”. Estudios $\lambda a m b d a$. Teoría y práctica de la didáctica en lengua y literatura 1 (1): 215-234. https://doi.org/10.36799/el.v1i1.32

Salado-Rodríguez, Lilián Ivetthe y Alberto Ramírez-Martinell. 2018. "Capital cultural en el contexto tecnológico: consideraciones para su medición en la educación superior". Revista iberoamericana de educación superior 9 (24): 125-137. https://doi.org/10.22201/iisue.20072872e.2018.24.268

SEP (Secretaría de Educación Pública). 2021. “¿Qué hacemos?” México: Subsecretaría de Educación Superior (SES). Fecha de consulta: 20 de febrero de 2021. https://www.educacionsuperior.sep.gob.mx/hacemos.html

Vega Falcón, Vladimir. 2017. "Una mirada al concepto de Capital Intelectual”. Revista Uniandes Episteme 4 (4): 491-503.

https://core.ac.uk/download/pdf/235987869.pdf

Verón Lassa, José Juan. 2016. “Ciudadanía y sociedad de la información: la tarjeta de Zaragoza”. Opción 32 (12): 877-899. https://www.redalyc.org/articulo.oa?id=31048903042

Virkus, Sirje y Anmar Salman. 2020. "Effective leadership behaviours and information culture in the higher education institution". Global Knowledge, Memory and Communication 70 (4/5): 418-441. https://doi.org/10.1108/GKMC-08-2020-0106 
Yong Amaya, Linda Evelyn, José Antonio Rodríguez Samaniego y Frida Ruso Armada. 2017. "El capital intelectual como factor de innovación y de impacto social en las universidades. Una mirada al Ecuador”. Espacio Abierto 26 (4): 205-219. https://www.redalyc.org/articulo.oa?id=12264368011

Zheng, Yingqin. 2005. "Information culture and development: Chinese experience of e-health", en Proceedings of the 38th Annual Hawaii International Conference on System Sciences, 1-11.

https://ieeexplore.ieee.org/document/1385545

Para citar este texto:

Caudillo, Dora, Socorro Encinas-Grijalva, Rubén F. Martínez-Rocha y Jesús Lau. 2022. "Cultura de la información en el contexto educativo universitario: aportes teóricos”. Investigación Bibliotecológica: archivonomía, bibliotecología e información 36 (90): 133-149.

http://dx.doi.org/10.22201/iibi.24488321xe.2022.90.58480 